\title{
Body Integrity Identity Disorder (BIID): Befragung von Betroffenen und Erklärungsansätze
}

\author{
Body Integrity Identity Disorder (BIID): Interrogation of Patients \\ and Theories for Explanation
}

Autor

Institut
E. Kasten

Institut für Medizinische Psychologie, Universität zu Lübeck

\author{
Schlüsselwörter \\ - BIID \\ - Body Integrity Identity \\ Disorder \\ - Amputee Identity Disorder \\ - Apotemnophilie \\ - Transabilität \\ - Amputation \\ - Amputierte \\ Key words \\ - BIID \\ - body integrity identity \\ disorder \\ - amputee identity disorder \\ - apotemnophilia \\ - transability \\ - amputation \\ - amputee
}

\section{Zusammenfassung \\ $\nabla$}

Apotemnophilie, Amputee Identity Disorder oder Body Integrity Identity Disorder (BIID) bezeichnen das intensive Gefühl, der Körper wäre „kompletter" nach der Amputation eines Gliedes. Der Artikel diskutiert die Frage nach übereinstimmenden Persönlichkeitseigenschaften Betroffener und fragt nach Motiven. Anhand von Aussagen von 9 Personen werden Beispiele für auslösende Erfahrungen zitiert. Im Gegensatz zu anderen Kindern waren die Betroffenen oft vom Anblick eines Behinderten fasziniert. Im Artikel wird untersucht, ob das entsprechende Körperteil häufiger Erkrankungen zeigte. Geschildert wird typisches Pretending-Verhalten. Geprüft wird, ob es Parallelen zu körperdysmorphen Erkrankungen (BDD), Fetischismus oder Wahn gibt. Diese fielen gering aus, in der Mehrzahl ist der Wunsch auf ein spezifisches Körperteil fixiert, die Betroffenen sind sich der Absonderlichkeit ihres Begehrens völlig bewusst und hadern mit dem Pro und Kontra. Sexuelle Motive fanden sich bei etwa einem Drittel. Einige der Befragten befanden sich in ärztlicher oder psychologischer Behandlung; dies brachte den Amputationswunsch nicht zum Verschwinden. Bei mehreren der unter BIID-Leidenden wechselte der Amputationswunsch von einer Seite auf die andere. Dieser Befund ist mit der gängigen Theorie einer ZNS-Läsion nicht erklärbar. Diese Menschen haben eher das Ideal eines „perfekten“ Körpers, der einen Arm oder ein Bein nicht umfasst. Die meisten bewundern die Schönheit eines Stumpfes und sehen Amputierte als „Helden“, die trotz der Behinderung ihr Leben meistern. BIID ist keine einheitliche Störung, sondern man muss mindestens 3 Achsen trennen: 1 . Stärke der neuronalen Dysfunktion, 2. psychische Komponenten (z.B. sekundärer Krankheitsgewinn) und 3. Intensität des sexuellen Interesses.

\section{Abstract \\ $\nabla$}

Apotemnophilia, Amputee Identity Disorder or Body Integrity Identity Disorder (BIID) is the intensive feeling that the body will be "more complete" after amputation of a limb. The article disputes the question of matching personality characteristics of these subjects and asks for motives. Based on reports of nine individuals, triggering experiences are referred. In contrast to other children, often these subjects were fascinated by the sight of a handicapped person. In the article is investigated, whether the concerned limb showed more affections. Described is typical pretending behavior. Parallels to body dysmorphic disorder (BDD), fetishism, or delusions are investigated. These were minor, in most cases the wish was fixated on a specific limb, the subjects were aware of the abnormity of their desire and quarreled with the pros and cons. Sexual motives were found in one third. Some of the interviewed persons were in medical or psychological therapy; this did not let the desire disappear. In several BIID sufferers the wish for amputation changed, e.g. from the left to the right leg. This finding is not in accordance with the brain-dysfunction-theory. These people rather have an ideal of a "perfect" body minus one arm or leg. Most admire the beauty of a stump, and see amputees as "heroes" who still master their life in spite of their handicap. BIID is not a homogenous disturbance, one should separate three axes: 1. Strength of neuronal dysfunction, 2. Psychic components (e.g. secondary morbid gain) and 3. Intensity of sexual interests.
Korrespondenzadresse Prof. Dr. phil. Erich Kasten Institut für Medizinische Psychologie, Universität zu Lübeck Ratzeburger Allee 160 23538 Lübeck erikasten@aol.com 


\section{Einleitung}

\section{$\nabla$}

„Die Seele fühlt sich zu einem Körper mit nur einem Bein (...) gehörig. Der Körper entspricht dieser inneren Wirklichkeit nicht“", schrieb ein Betroffener. Aus bislang völlig unbekannten Gründen empfinden manche Menschen ein Glied nicht als Teil des Selbst. Dieses Gefühl kann so intensiv sein, dass die Betroffenen eine Amputation anstreben oder, wenn das Gesundheitssystem dies verweigert, selbst versuchen, sich einen Finger, Arm oder ein Bein zu entfernen. Diese Personen sind der festen Überzeugung, dass ihr Körper erst nach einer Amputation ihrem mentalen Körperbild entspricht. Von Money u. Mitarb. [37] wurde diese Störung zunächst als „Apotemnophilie“ bezeichnet („Freund des Abschneidens“); Furth u. Mitarb. [23] bzw. Smith [48] vergaben die Bezeichnung „Amputee Identity Disorder“ (AID). Heute gibt man meist der Bezeichnung BIID („Body Integrity Identity Disorder") den Vorrang [20]. Die Betroffenen bezeichnen sich selbst als „Wannabe“ (von engl. want to be). Mitunter wird im Vorfeld versucht, durch Gebrauch von Krücken (bei hochgeschnürtem Bein), Prothese oder Rollstuhl ein Gefühl der erwünschten Beeinträchtigung zu erzeugen („pretending“). Bei einem Teil ist auch eine erotische Komponente vorhanden, dann werden Menschen mit sichtbaren Behinderungen als sexuell attraktiv empfunden (Amelotismus, Akrotomophilie, bzw. „devotee“) oder die Betroffenen erregen sich selbst durch die Vorstellung des eigenen, amputierten Körpers [22].

Bis zur Jahrtausendwende gab es nur ein rundes Dutzend Arbeiten, die sich mit dem Phänomen beschäftigten $[9,11,18,25,37$, $38,42,45,50,52]$. Durch Presseberichte und insbesondere durch Internet-Foren hat das Phänomen inzwischen breite Aufmerksamkeit erfahren, so dass sich seit der Jahrtausendwende auch die Anzahl wissenschaftlicher Arbeiten potenziert hat [1,3,5,6, $8,10,14,15,17,20,22-24,29,31,36,39-41,43,49]$.

Die bislang breiteste Untersuchung stammt von First [20], der strukturierte Telefon-Interviews mit 52 Betroffenen durchführte. $17 \%$ hatten eine Amputation, hiervon war immerhin ein Drittel von Ärzten durchgeführt worden. First wies darauf hin, dass der Amputationswunsch keiner akuten psychotischen Störung entstammte; nach seiner Einschätzung wirkte keiner der Befragten wahnhaft. Bei nahezu allen bestand der Wunsch nach Amputation schon seit früher Kindheit. Dies unterscheidet BIID-Betroffene stark von Psychotikern, die sich z.B. im Verlauf eines akuten Schubes eine Hand, die Brust oder den Penis abschneiden bzw. sich selbst blenden $[12,13,26,31,34,47]$. Aktuell arbeitet First daran, BIID in die neue Auflage des DSM aufzunehmen. Bislang gibt es kaum Studien, in denen die Möglichkeiten medikamentöser oder psychotherapeutischer Intervention systematisch geprüft wurden. Die wenigen Arbeiten (meist Einzelfallstudien) zeigten nur begrenzte Effekte auf die Stimmung der Patienten und ihren Umgang mit dem drängenden Wunsch nach Amputation; dieser verschwand jedoch weder durch Antidepressiva noch durch kognitive Verhaltenstherapie $[1,8,29]$.

Mediziner weigern sich in der Regel, ein gesundes Körperteil zu amputieren, denn derzeit gibt es eine Fülle ethischer und juristischer Probleme. Im Jahr 2000 führte der schottische Arzt Dr. Robert Smith 2 Beinamputationen bei Patienten mit BIID durch. Nach einem Bericht des Fernsehsenders BBC verbot die britische Ärztekammer weitere Amputationen $[3,16]$. Im $\S 226 a$ des deutschen STGB heißt es hierzu: „Wer eine Körperverletzung mit Einwilligung des Verletzten vornimmt, handelt nur dann rechtswidrig, wenn die Tat trotz der Einwilligung gegen die guten Sitten verstößt." Die hierzu entfachte juristische Diskussion ist bis- lang zu keiner eindeutigen Schlussfolgerung gekommen $[10,36,41,43,5]$. Vergleiche wurden u.a. mit Tattoos, Piercings, Geschlechtsumwandlungen und alle Formen der SchönheitsChirurgie angestellt, die auf der Grundlage der psychischen Unzufriedenheit des Betroffenen letztlich alle „Körperverletzungen mit Einwilligung" sind. Sie erzeugen aber im Gegensatz zu BIID keine bleibende Behinderung. Ryan [45] verwies auf das Recht zur autonomen Entscheidung und verglich das Verlangen nach Amputation mit Bungee Jumping, Verweigerung der Bluttransfusion bei den Zeugen Jehovas oder Euthanasie.

Die Betroffenen leiden psychisch oft erheblich unter ihrem Wunsch und legen in etlichen Fällen schließlich selbst Hand an. Ein Patient schoss sich selbst ins Knie, um die Ärzte zu zwingen die Amputation durchzuführen; andere benutzten selbstgebaute Mini-Guillotinen, elektrische Sägen, Erfrierungen durch Trockeneis, bewusst erzeugte Infektionen oder legten ihr Bein vor einen einfahrenden Zug [17,22,29,31,37]. Aktuell suchen diejenigen mit ausreichenden finanziellen Ressourcen einen Arzt in einem Land der Dritten Welt und benutzen dann die Ausrede eines Unfalls. Dies verursacht eine Fülle versicherungsrechtlicher Probleme, da die Betroffenen oft auch später noch hohe Behandlungskosten verursachen, meist eine private Unfallversicherung besitzen und mitunter arbeitsunfähig werden.

Bridy [10] und Bayne \& Levy [1] äußerten die Ansicht, solange es keine sinnvolle Behandlungsmöglichkeit für die Betroffenen gibt, sei die chirurgische Amputation im Krankenhaus letztlich das kleinere Übel. Bruno [11], Riddle [44] und Müller [39,40] weisen dies zurück. Müller äußerte die Ansicht, dass es irgendwann eine Behandlung geben könnte und dass man den Betroffenen daher die Amputation verweigern solle, damit diese Patienten eines Tages nicht nur leidens-, sondern auch behinderungsfrei leben können. Bislang gibt es kaum Untersuchungen an Betroffenen, die eine Amputation erfolgreich durchgeführt haben. Nach Angaben von First [20] und Bayne und Levy [1] sind diese Personen zufriedener, streben keine weiteren Amputationen an und leiden nur selten unter Phantomschmerzen.

BIID-Betroffene können gewöhnlich keine wirklich sinnvolle Begründung ihres Wunsches liefern, er ist ihnen selbst auch unheimlich. Viele machen Stadien durch, in denen sie fürchten, das Begehren sei ein Zeichen, dass sie beginnen „wahnsinnig“ zu werden. Phasenhaft kann der Wunsch so stark werden, dass er kaum noch erträglich ist [29]. Im Gegensatz zu Psychotikern wissen alle, dass ihr Begehren nicht normal ist und von der Umwelt nicht toleriert wird. Daher ist kaum jemand in der Lage mit anderen hierüber zu sprechen; selbst bei Fachleuten stoßen sie überwiegend auf Unverständnis. Der wesentlichste Gedankenaustausch läuft in pseudonymisierter Form über Internet-Foren. Die Mitglieder berichten übereinstimmend, dass es ihnen geholfen hat festzustellen, dass sie mit dieser Störung nicht alleine sind [6]. Abgesicherte Zahlen über die Häufigkeit von BIID gibt es bislang nicht. Horn [29] schätzte die Prävalenz auf 1-3\% der „klinischen Population“. Müller [39,40] und Bayne \& Levy [1] schätzten die Gesamtzahl auf „mehrere Tausend weltweit“. Im April 2008 hatten einschlägige Internet-Foren folgende Mitgliederzahlen: 1723 (Yahoo „fightin-it“), 561 („need2be1“), 591 („BIID and Admirers Circle of Friends“), bzw. 358 („the biid affair“). 


\section{Auffälligkeiten des Persönlichkeitsprofils $\nabla$}

Die Ursachen sind unbekannt. Die aktuelle Literatur diskutiert, ob BIID die Folge einer psychischen Störung sein könnte, genannt wurden z. B. Persönlichkeits-, körperdysmorphe oder neurotische Störungen, Depression, Wahn oder Psychosen. In einer eigenen Studie wurde versucht zu erfassen, ob und in welchem Ausmaß BIID-Betroffene tatsächlich psychische oder psychiatrische Abweichungen zeigen (Kasten, subm.). Hierfür konnten 7 Personen untersucht werden, die diverse standardisierte psychologische Fragebogen ausfüllten: FBL-R („Freiburger Beschwerde Liste - Revision“, [19]), Gießen-Test [4], Becks Depressions-Inventar [27], TIPI („Trierer Integriertes PersönlichkeitsInventar“ [2]), SCL-90-R („Symptom Checkliste“ [21]) und der „Rosenzweig Picture Frustration Test“ (PFT [28]). Darüber hinaus kam ein selbstentwickelter Fragebogen zur Anwendung, der Fragen hinsichtlich Entstehung, Art und Auswirkungen des Amputationswunsches enthielt. Allen Befragten wurde absolute Anonymität und Vertraulichkeit zugesichert. Insgesamt lagen die Persönlichkeitsprofile fast aller Tests im durchschnittlichen Bereich. Lediglich in der Symptom-Checkliste (SCL-90R) war der Psychotizismus-Wert leicht erhöht (T-Wert 60,5). Entgegen aller Annahmen zeigte der Wert von 8,1 im Becks Depressionsinventar gar keine Hinweise auf erhöhte Depressivität. Lediglich die Freiburger Beschwerdeliste (FBL-R) hatte einen insgesamt angehobenen Kurvenverlauf, überwiegend im oberen Durchschnittsbereich. Der Test dient der Untersuchung somatischer Beschwerden, insbesondere die Skala „emotionale Reaktivität“ (d. h. psychosomatische Reaktionen auf emotional belastende Situationen) war bei allen Befragten erhöht. Lediglich einer der 7 Teilnehmer dieser Studie zeigte psychopathologische Auffälligkeiten und einen massiv erhöhten Depressionswert. Die Daten untermauern den Befund anderer Arbeiten, dass BIID sich nicht auf eine bekannte psychische Störung zurückführen lässt.

\section{Äußerungen von BIID-Betroffenen \\ $\nabla$}

Aufbauend auf den Daten der ersten Studie konnten vom Verfasser inzwischen 9 Betroffene aus dem deutschsprachigen Raum mithilfe eines selbstentwickelten Fragebogens befragt werden. Eine Kurzcharakteristik der Personen findet sich in Tab. 1.

\section{Motive}

Die Teilnehmer dieser Befragung können kaum rationale Beweggründe für den Amputationswunsch nennen, die Argumente lagen eher auf einer emotionalen Ebene. So schrieb z. B. der Befragte-1: „Ich weiß es wirklich nicht. Ich will es ganz einfach. Ich fühle, wo der Stumpf in meinen Oberschenkeln enden würde und habe das Gefühl einer starken ,Begierde‘ (...) mit 2 Oberschenkelstümpfen zu leben.“ Proband-2: „Ich fühle einfach, dass mein linkes Bein ,zuviel‘ ist, dass es nicht da sein sollte, sondern stattdessen nur ein Stumpf. (...) Es gehört nicht zum wahren Bild meines Körpers. Aber ich finde es nicht abstoßend und hasse es auch nicht.“ Proband-3: „1. Nie mehr ohne Krücken auch nur einen Schritt laufen zu können. 2. Dieses elegante leichte Schwingen zwischen den Krücken. 3. Eine grenzenlose Neugier zu erfahren wie es ist, amputiert zu sein. 4. Das zufriedene Gefühl, das ich beim Pretenden erfahre - und das Wissen, dass ich es einbeinig packen werde. 5. Die Herausforderung anzunehmen und zu bestehen. 6. Die leichtere Möglichkeit, andere Amputierte kennenzulernen. 7. Die Asymmetrie des eigenen
Körpers wahrzunehmen.“ Pb.-4: „Gefühl der Nichtzugehörigkeit des linken Beins zum restlichen Körper. Gefühl, ohne das überzählige Bein im richtigen Körper zu sein. Vorhandensein des Beins stört, quält, erzeugt seelisches Ungleichgewicht. (...) Spüre genau die Linie im Oberschenkel, wo das gefühlte Bein endet (seit Jahren stabil).“ Pb.-5: „Das Bein/der Finger wirken wie nicht zum Körper zugehörig. Sie sind zwar da, würden aber auch nicht fehlen, wenn sie weg wären. Die Amputation würde den Körper komplettieren. Erst dann wäre er, wie er sich anfühlt/sein soll.“ Pb.-6: „Es ist kein Wunsch, es ist in mir ... Das ist so, wie wenn man heterosexuell oder homosexuell ist - ein Teil der Persönlichkeit.“ Pb.-8: „Realisierung meiner Identität (um mich endlich seelisch und körperlich besser zu fühlen als in den letzten 30 Jahren - körperliche und seelische Ganzheit).“

\section{Pretending}

Fast alle Betroffenen neigen zu Ersatzverhalten („pretending“), das auch dazu dient auszuprobieren, ob man mit dem Zustand einer Behinderung zurecht kommen würde. So setzte sich der Befragte-1 mit gewünschter Doppelamputation hin und wieder auf den Fußboden und versuchte einige Meter auf seinen Händen zu laufen. Der Betroffene-2 band sein abgewinkeltes Bein nach hinten und benützte Krücken, um eine Amputation auszuprobieren. Der Befragte-3 steckte sein abgewinkeltes Bein in ein Hosenbein, zog einen langen Mantel an und ging dann mit 2 Krücken in die Stadt: „Als 'Pretender' (zu Hause und auch unterwegs) habe ich schon Etliches erledigt (Einkaufen, Wäsche bügeln, sogar Stall ausmisten...) und Reaktionen meiner Umwelt auf mich als Krüppel getestet und ich weiß, dass es klappen wird.“ Ähnliche Verhaltensweisen zeigten auch alle anderen Befragten.

\section{Auslösende Kindheitserfahrungen}

Während die meisten Kinder eher mit Erschrecken und Mitleid auf den Anblick eines Amputierten reagieren, finden sich bei vielen Betroffenen schon in früher Kindheit stark positiv-getönte emotionale Reaktionen: „Es gab da einen Jungen, der ein Bein in so einer Schiene aus schwarzem Metall mit Lederriemen hatte und ziemlich hinkte. Den fand ich toll. (Pb.-1). „Als ich etwa 10 war, erzählte mein Friseur, dass sein Kollege (...) bei einem Motorradunfall ein Bein verloren hatte - ich war wie elektrisiert und zeichnete danach noch Wochen später Männchen mit nur einem Bein.“ (Pb.-6). „Außerdem blieben mir unauslöschlich 2 ähnliche Situationen aus derselben Zeit im Gedächtnis; am Strand robbte ein gleichaltriger Junge ins Wasser, rechts unterschenkelamputiert. Da er nur mit Badehose bekleidet war, sah ich erstmals einen Stumpf pur. Einen anderen, ebenfalls gleichaltrigen Jungen, links unterschenkelamputiert, lernte ich (ohne Prothese) flüchtig kennen. (...) Ich fuhr oft mit dem Fahrrad in jene Straße wo er wohnte. Dies in der Hoffnung, ihm zu begegnen und unter irgendeinem Vorwand ansprechen zu können. (...) Bald nach dem Kriege, ich war höchstens 10 Jahre alt, da standen noch die Ruinen. (...) In einer der Höhlungen saß ein Junge, nicht viel älter als ich, der verkaufte irgendetwas. Er war beidseitig oberschenkelamputiert. Wenn ich später dort vorbeiging, hoffte ich immer ihn zu sehen.“ (Pb.-7). „1974 war ich 8 Jahre alt. An einem schönen späten Sommernachmittag in Köln fiel mein Blick kurz und unvermittelt auf einen jungen Mann im Rollstuhl. Bis heute fühle ich mich in einer unerklärbaren Weise von ihm angezogen. Vergeblich versuchte ich damals meinen Vater mit einem Vorwand dazu zu bewegen, gemeinsam mit mir ihm zu folgen. Unauslöschlich brannten sich seine dünnen 
Tab.1 Kurzcharakteristik der befragten 9 Betroffenen.

\begin{tabular}{|c|c|c|c|c|c|c|c|}
\hline Nr. & Geschl & Alter & $\begin{array}{l}\text { Ausbildung / } \\
\text { Beruf }\end{array}$ & $\begin{array}{l}\text { Sex. } \\
\text { Orientierung }\end{array}$ & $\begin{array}{l}\text { Krankheiten, Ope- } \\
\text { rationen }\end{array}$ & $\begin{array}{l}\text { Amputations- } \\
\text { Wunsch }\end{array}$ & $\begin{array}{l}\text { Amput.-Wunsch } \\
\text { seit Alter ca. }\end{array}$ \\
\hline 1 & $\mathrm{~m}$ & Anf. 40 & Abitur/Freiberufler & $\begin{array}{l}\text { Homosex., } \\
\text { mit Mann }\end{array}$ & $\begin{array}{l}\text { Hüftgelenkserkran- } \\
\text { kung in der Kindheit }\end{array}$ & $\begin{array}{l}\text { Beide Beine ab } \\
\text { Oberschenkel }\end{array}$ & $7 / 8 \mathrm{~J}$ \\
\hline 2 & $\mathrm{~m}$ & Ende 30 & Abitur/Berater & $\begin{array}{l}\text { Heterosex., } \\
\text { mit Frau }\end{array}$ & $\begin{array}{l}\text { Arthroskopie, } \\
\text { Depressionen }\end{array}$ & $\begin{array}{l}\text { Linkes-rechtes Bein } \\
\text { (wechselnd) ab } \\
\text { Mitte Oberschenkel }\end{array}$ & $4 / 5 \mathrm{~J}$ \\
\hline 3 & $\mathrm{~m}$ & Mitte 40 & $\begin{array}{l}\text { Abitur/Projekt- } \\
\text { Management }\end{array}$ & $\begin{array}{l}\text { Bisex., } \\
\text { mit Frau + Kind }\end{array}$ & $\begin{array}{l}\text { Variozele-Opera- } \\
\text { tion, Armbruch }\end{array}$ & $\begin{array}{l}\text { Linkes Bein ab Mitte } \\
\text { Oberschenkel }\end{array}$ & $6 \mathrm{~J}$. \\
\hline 4 & $\mathrm{~m}$ & Anf. 40 & $\begin{array}{l}\text { Abitur/Transport- } \\
\text { Kaufmann }\end{array}$ & Homosex., Single & $\begin{array}{l}\text { Meningitis als Kind, } \\
\text { Gallenstein-Opera- } \\
\text { tion }\end{array}$ & $\begin{array}{l}\text { Ein Bein ab Mitte } \\
\text { Oberschenkel }\end{array}$ & $10 \mathrm{~J}$. \\
\hline 5 & $\mathrm{~m}$ & Anf. 30 & Abitur/Betriebswirt & $\begin{array}{l}\text { Homosex., } \\
\text { mit Mann }\end{array}$ & $\begin{array}{l}\text { Beinbruch mit } 3 \text { J., } \\
\text { Schuppenflechte, } \\
\text { gelegt. Schmerz re. } \\
\text { Knie, Beschneidung } \\
\text { d. Penis }\end{array}$ & $\begin{array}{l}\text { Rechtes Bein ab } \\
\text { Mitte Oberschenkel } \\
\text { und rechter Zeige- } \\
\text { finger }\end{array}$ & $8-10 \mathrm{~J}$ \\
\hline 6 & $\mathrm{~m}$ & Mitte 40 & $\begin{array}{l}\text { Abitur } \\
\text { Geologe }\end{array}$ & $\begin{array}{l}\text { Homosex., } \\
\text { mit Mann }\end{array}$ & $\begin{array}{l}\text { Hodenhochstand, } \\
\text { Mandel-OP, Entzdg. } \\
\text { Schleimbeutel Knie, } \\
\text { rechts }\end{array}$ & $\begin{array}{l}\text { Zunächst linkes, } \\
\text { dann rechtes Bein } \\
\text { ab Mitte Ober- } \\
\text { schenkel }\end{array}$ & $8 \mathrm{~J}$. \\
\hline 7 & $\mathrm{~m}$ & Anf. 70 & $\begin{array}{l}\text { Abitur, Medien- } \\
\text { branche }\end{array}$ & $\begin{array}{l}\text { Homosex., } \\
\text { mit amput. Mann }\end{array}$ & $\begin{array}{l}\text { Poliomyelitis als } \\
\text { Kind, Verkehrsunfall } \\
\text { mit } 50 \text { J., Blasen- } \\
\text { karzinom-Op. } 60 \text { J., } \\
\text { Fazialis-Lähmung, } \\
\text { Blutdruck, } \\
\text { Erstickungsanfälle }\end{array}$ & $\begin{array}{l}\text { Zunächst linkes } \\
\text { Bein, später beide } \\
\text { Beine, zuletzt linkes } \\
\text { Bein. }\end{array}$ & $10-12$ J. \\
\hline 8 & $\mathrm{~m}$ & Mitte 40 & $\begin{array}{l}\text { Abitur, Unterneh- } \\
\text { mer }\end{array}$ & $\begin{array}{l}\text { Heterosex., } \\
\text { mit Frau }\end{array}$ & $\begin{array}{l}\text { gelegentl. Dereali- } \\
\text { sationsphänomene, } \\
\text { Magen-Darm- } \\
\text { Probleme }\end{array}$ & $\begin{array}{l}\text { Querschnittsläh- } \\
\text { mung beider Beine }\end{array}$ & $8 \mathrm{~J}$ \\
\hline 9 & $\mathrm{~m}$ & Mitte 40 & FHS-Reife, Meister & $\begin{array}{l}\text { Heterosex., } \\
\text { mit Frau }\end{array}$ & Mandel-Op. & $\begin{array}{l}\text { Linkes Bein, } 10 \mathrm{~cm} \\
\text { über Knie }\end{array}$ & $8 \mathrm{~J}$. \\
\hline
\end{tabular}

Beine, der kerzengerade Rücken, dessen oberer Anteil auf dem unteren sich bei jeder Regung auszubalancieren schien und seine starken Oberarme in mein Gedächtnis ein. Sein Oberkörper schien ein Dreieck zu bilden aus dem kurzen muskulösen oberen Bereich und den gedachten Schenkeln, die am offensichtlich gelähmten unteren Teil seines Rumpfs hätten gezogen werden können. Das kubistisch anmutende Bild des jungen Mannes war unvorhergesehen und vom ersten Augenschein an in mir zum Idealbild für meinen eigenen Körper geworden.“ (Pb.-8).

Diese starke emotionale Reaktion bleibt offenbar lebenslang erhalten. Ausgehend hiervon ist es nicht verwunderlich, dass die Betroffenen häufig schon als Kinder einen Behinderten spielten, z. B.: „Irgendwann in meiner Kindheit (...) spielte ich mit Freunden Robin Hood, und weil ich im Fernsehen gesehen hatte, dass einer dabei war bei seiner Truppe, dem der Sheriff ungerechterweise wegen Diebstahls die Hand abhacken ließ, zog ich mir einen Socken drüber und spielte den. (Ich würde doch zu gern wissen, ob den anderen auffiel, was das für mich bedeutete.) Dann spielte ich mal mit einer Schulfreundin im elterlichen Keller amputiert. Ich bastelte Krücken (sehr behelfsmäßig) und wir gingen damit rum.“ (Pb.-1). „Ich habe ca. mit 8 Jahren festgestellt, dass ich es als angenehm empfinde, wenn ich mir mein linkes Bein abgebunden habe und das Gefühl aus dem abgebundenen Teil des Beines verschwunden war. Selbst der Schmerz, der dadurch erzeugt wurde, war besser als sein Bein zu spüren. Ich habe das über einen relativ langen Zeitraum gemacht, bis ich erfahren habe welche Gefahren damit verbunden sind. Danach habe ich das eingestellt, ich wollte mich ja damit nicht umbringen. Dann habe ich über Jahrzehnte versucht mich von diesen Gedanken zu lösen, weil ich dachte ich wäre der einzige mit dieser verrückten Idee." (Pb.-9).

\section{Erkrankungen des Körperteils}

Weitgehende Verbreitung hat die Theorie eines entwicklungspsychologischen Ansatzes gefunden, wonach schon zu einem frühen Zeitpunkt der kindlichen Entwicklung eine Störung des Körperschemas entsteht. Hierfür soll sprechen, dass sich anamnestisch oft eine Erkrankung des Körperteils in einer frühen Entwicklungsperiode nachweisen lässt [39]. An Erkrankungen in der Kindheit wurde von dem Befragten-1 eine Hüfterkrankung in der Kindheit genannt, die nach 6 Wochen wieder vorüberging und von Proband-5 ein Beinbruch im Alter von 3 Jahren (unbekannt, welches Bein). Von den 9 Befragten hatten 3 im späteren Leben chronische Beschwerden des Beines, die z. T. medizinische Eingriffe nötig gemacht hatten (Pb.-2: Arthroskopie des rechten Kniegelenks, Pb.-5 Zysten in den Knien mit Schmerzen des rechten Knies und Pb.-6: Schleimbeutelentzündung im Knie, Bänderüberdehnung, häufige Schmerzen der Achillessehne). Als alleiniger Grund für die Amputationsbegierde kommen solche somatischen Krankheiten aber sicherlich nicht in Betracht. Wie weiter unten erörtert wird, spielen sie keine Rolle dabei ob, sondern nur welches Körperteil amputiert werden soll. Eine Person litt in der Kindheit unter Poliomyelitis, eine andere gab eine 
Hirnhautentzündung an, wodurch sich die Frage nach hirnorganischen Grundlagen stellt.

\section{Eine ZNS-Läsion als Ursache?}

Bei allen Teilnehmern dieser Studie entstand der Wunsch nach einer Behinderung schon in der Kindheit oder frühen Jugend. Man vermutet daher eine ZNS-Schädigung zu einem sehr frühen Zeitpunkt, z.B. eine Läsion im Bereich des somatosensorischen Kortex, wodurch sich ein Körperbild entwickelt hat, das ein Gliedmaß nicht umfasst [7,39]. Blanke und Mitarbeiter [7] vermuten aufgrund einer Befragung von 20 Betroffenen eine frontoparietale Dysfunktion, da 65\% der Teilnehmer anomale Gefühlssensationen im entsprechenden Körperteil hatten. Für diese Theorie spricht, dass viele Betroffene schon vor einer Amputation das Stumpfende sehr exakt fühlen können: „Bei mir selber ist das Überraschende, dass ich seit einiger Zeit nicht nur die Linie, sondern sogar die Form der Stümpfe spüre, wie sie in meinen Oberschenkeln verborgen sind, sehr konkret sogar, und häufig morgens in der Zeit, wo ich nicht mehr ganz schlafe und noch nicht wach bin.“ (Pb.-1). ,... ich weiß aber genau, wo der Stumpf laufen soll und wie lang und formschön der Stumpf auszusehen hat“ (Pb.-3). „Seit einiger Zeit spüre ich deutlich, wo das Bein nach einer Amputation enden würde“ (Pb.-6). „...es ist eine genaue Amputationshöhe, die ich körperlich spüre (...) Wenn ich mein linkes Bein berühre ist es auch ein ganz anderes Gefühl als würde ich mein rechtes Bein berühren." (Pb.-9)

Allerdings müsste es bei einer ZNS-Läsion sensomotorische Ausfälle des betreffenden Körperteils geben. Zumindest ein Teil der Betroffenen trieb Sport (z.B. Jogging) in erheblichem Ausmaß, was damit nicht kompatibel wäre. Der Theorie einer Läsion des parietalen sensorischen Kortex widerspricht insbesondere, dass der Amputationswunsch sich bei einigen der hier Untersuchten ausweitete oder von einem Körperteil auf ein anderes wechselte. Beginnend in seiner Kindheit bis 2002 wünschte der Betroffene-2 sich eine Amputation des linken Beines. 2002 und 2003 fokussierte sich dieser Wunsch auf das rechte Bein und wechselte dann ab 2003 erneut auf das linke Bein. Ursache für diesen Wechsel waren Überlegungen nach einer Knie-Operation: „... dass es ja ziemlich unklug wäre, nicht das kranke Bein zu amputieren.“ [32]. Der Befragte-3 war zunächst unentschieden, welches Bein amputiert werden sollte. Später wurde der Wunsch spezifischer und er wollte eine Entfernung des linken Beins ab der Mitte des Oberschenkels. Als Begründung schrieb er: „Ich kann für mich noch einmal betonen: Es geht mir nicht darum, mein linkes Bein loszuwerden. Ich will einbeinig werden, und das linke ist einfach weniger notwendig als das rechte (z.B. beim Autofahren). Das ist das ganze Geheimnis meiner Seitenwahl.“ Der fünfte Befragte wünschte sich zunächst eine doppelte Arm-Amputation, etwa ab 15/16 Jahre die Entfernung des rechten Beins, ab 21/22 Jahre dann des rechten Beins und des rechten Zeigefingers. Der Befragte-6 wünschte sich, beginnend in seiner Jugend, eine Entfernung des linken Beins. Im Dezember 2005 verlagerte sich dies auf das andere Bein, da er dort vermehrt Schmerzen der Achillessehne spürte.

Diese Verlagerung des Amputationswunsches aus rein rationalen Gründen scheint nicht selten zu sein. Ein erfolgreich amputierter US-Amerikaner schrieb: "I had always wanted to be an Above the Knee amputee, but I mostly wanted to be a Right Above Knee amputee. However, three months before my scheduled amputation, I developed a 'trick' left knee and decided that it would be ridiculous to have my right leg amputated with a 'trick' left knee because I would spend most of my time on the floor from falling. So, I had my left leg amputated instead and I have been fine with it."

Andererseits ist es für die Betroffenen keinesfalls gleichgültig, welche Behinderung sie bekommen. Der Wunsch ist stark auf ein bestimmtes Körperteil fixiert und wechselt offenbar nicht auf ein beliebiges anderes, sondern bestenfalls von einer Körperseite auf die andere. So schrieb der Befragte-1 z.B.: „Sie werden lachen, ich zittere um jeden Zahn, um den sich meine Zahnärztin bemüht, und ich fände es den reinen Horror, wenn ich etwa blind wäre oder taub oder einen Arm verlöre. Oder noch schlimmer: wenn irgendwas gelähmt wäre.“

\section{Gibt es Parallelen zu körperdysmorphen Störungen?}

Patienten mit körperdysmorphen Störungen („body dysmorphic disorder“, BDD) empfinden bestimmte Körperteile als hässlich und wünschen daher eine operative Veränderung. Keiner der hier befragten BIID-Betroffenen äußerte eine negative Beurteilung des Körperteils, welches amputiert werden sollte. So schrieb der Befragte-1 hinsichtlich der Beine habe er keinerlei negative Gefühle, diese seien weder krank noch empfinde er sie als hässlich. Er habe lediglich ein Gefühl seine Beine seien weniger „beseelt“ als andere Körperteile. Der Befragte-3 schrieb hierzu: „Bei mir ist es durchaus nicht so, dass ich mein linkes Bein hasse oder es als nicht zu mir gehörig erachte. Bei mir ist das Ziel 2 Krücken zu benötigen, ein Krüppel zu werden, das treibende Motiv. Die Amputation ist nur der einfachste Weg dahin zu kommen.“ Nr.-4 sagte aus: „Fühlt sich fremd, nicht zugehörig an. Ist aber völlig gesund und funktionstüchtig. Also benutze ich es auch normal, so lange es noch dranhängt." Proband-5 schrieb: „Von außen betrachtet wirkt es ,leicht verschwommen“. Das Bein fühlt sich innerlich betrachtet leicht pelzig an. Es ist zwar da, fühlt sich aber nicht als zugehörig an. Die Form aus einem Guss schließt Bein und Finger nicht ein, so wie ein Riss an einer Marmorstatue die Teile trennt.“ Befragter-6: „Ich sehe das als Erweiterung von Möglichkeiten meines Körpers und mit meinem Körper umzugehen. (...) Nach wie vor habe ich das (ziemlich!!) sichere Gefühl, ja die Gewissheit, dass es mir ohne Bein besser ginge - erheblich vermutlich und leistungsfähiger wäre ich auch (schon weil nicht so viel Zeit drauf ginge, über BIID nachzudenken...). (...) Es gibt Tage (zugegeben nicht jeder), da würde ich mich ohne Zögern und Überlegen unters Messer legen - und ich bin sicher, es wäre gut.“ Und Nr.-9: „Ich habe zu diesem Stück Bein ein anderes körperliches Empfinden als zu meinem Körper und allen anderen Körperteile. Ich würde niemals auf mehr als auf mein linkes Bein verzichten. Es gehört nicht zu meinem Körper. Wie schon ausgeführt, fühlt sich dieses Teil des Beines an, als würde es einem Fremden gehören. Als wenn ich einen Fremden an seinem Bein berühre. Selbst das Gefühl im linken Bein ist ein anderes Gefühl als in meinem rechten Bein.“

Während körperdysmorphe Patienten ihre äußere „Entstellung“ meist verbergen und sozialen Kontakt vermeiden, scheinen manche BIID-Betroffene demgegenüber zu planen, diese Behinderung bei erfolgreicher Amputation regelrecht vorzuzeigen. So schriebt der Befragte-3: „Im Gegensatz zu manchen Amputierten werde ich natürlich nicht versuchen, diesen körperlichen Mangel zu kaschieren, sondern meinen Stumpf bzw. meine Prothese so zeigen, dass sie für jeden sichtbar ist." Der Befragte-6 dagegen möchte auch nach der Amputation normal aussehen: „Ich würde als Amputierter auf jeden Fall eine Prothese wollen, denn ich will nicht für andere ,behindert' aussehen, weil ich mich ja selbst nicht so fühlen würde. Und hier kriege ich 
(manchmal) selbst nicht die Kurve: warum wünsche ich mir über einen Umweg - einen Zustand, den ich eigentlich jetzt schon habe?? (...) Es geht darum, zu wissen, amputiert zu sein, und damit den Körper zu haben, den man sich wünscht. Das schließt (für mich) absolut nicht aus, alle (Hilfs)Mittel zu nutzen, um wieder so mobil wie vorher zu sein."

\section{Ist BIID eine Form von Fetischismus?}

Noch immer herrscht Begriffsverwirrung zwischen BIID bzw. Apotemnophilie ohne erotisches Interesse und Amelotismus, Akrotomophilie, bzw. „devotee“ mit sexueller Komponente. Einzelne Berichte lassen den Verdacht aufkommen, dass hinter BIID lediglich eine spezielle Art von Fetischismus stecken könnte $[37,38]$. Allerdings kreuzten 4 der Befragten auf die Frage nach einer sexuellen Komponente eindeutig „Nein“ an und verneinten auch, sich einschlägiges Bildmaterial amputierter Personen im Internet oder in Heften anzusehen. Bei 2 der Befragten war das erotische Interesse untergeordnet. Hiervon schrieb der eine, er werde auch sexuell von Amputierten angezogen, aber nicht immer und nicht primär, der andere sagte: „Es gibt eine sexuelle Komponente, welche aber nur sehr gering ist. Ästhetisches Empfinden des asymmetr. Körpers als richtig, schön. Hatte vorher schon sexuellen Kontakt zu Amputierten; die Qualität dieser Kontakte war aber unabhängig von der Amputation. (...) Sex mit amp. Partner ist nicht besser oder schlechter als mit Nichtamputierten." Im Gegensatz hierzu hatten 3 der Befragten auch erhebliches erotisches Interesse an Amputierten. Teilnehmer-3 gab Motive an ,....die bei mir auch mit einer sehr starken erotischen Komponente gepaart sind. Diese erotische Komponente, die sich darin ausdrückt, dass ich nur dann sexuell erregt bin, wenn ich mich als gehbehindert vorstelle (Amputation, Kinderlähmung, ungleich lange Beine ...), hat mein Eheleben kaputtgemacht, da ich mit meiner Frau nichts mehr anzufangen weiß." Er surfte auch 2- bis 5-mal pro Woche zwischen 10 und $60 \mathrm{~min}$ auf entsprechenden Internet-Seiten. Auch der Befragte-5 fühlte sich von Amputierten sexuell angezogen, er besuchte mehrfach pro Woche einschlägige Internetseiten. Beide hatten jedoch noch nie Intimkontakt mit Amputierten. Besonders interessant ist die Lebensgeschichte des Befragten-7, der inzwischen mit einem oberschenkelamputierten Partner zusammenlebt: „Schon als 12-jähriger steckte ich die Beine kniegebeugt in die Turnhose, die Füße also in der Hose am Hintern. Ich hopste herum, stellte 2 Stühle links und rechts neben mich, legte die Hände darauf, drückte mich in die Höhe, so dass die Stümpfe schwebten und schaukelte hin und her. Das kostete viel Kraft. Und genau während dieser Kraftprobe kam das ,Gefühl', wie ich es damals nannte. Von Orgasmus hatte ich noch nie gehört." Und an anderer Stelle noch: „Es ist noch gar nicht lange her, da geschah Folgendes: Ich klingelte aus ganz anderen Gründen an einer mir bis dahin unbekannten Haustür. Es öffnete ein junger Mann - flott auf seinen Beinstümpfen laufend!!! Die sachliche Angelegenheit besprachen wir, dann fuhr ich nach Hause, kaum noch fahrtauglich. Ich geriet in einen unbeschreiblichen Zustand. Kaum zu Hause angekommen, hatte ich einen nicht enden-wollenden Orgasmus und blieb auch danach stundenlang in einem noch nie erlebten Schwebezustand.“

Denkbar wäre eine Konditionierung zwischen ersten sexuellen Erfahrungen in der Pubertät und Kontakt mit Behinderten. Allerdings zeigte nur ein Drittel der hier Befragten ein erotisches Interesse an Amputierten, zum anderen tauchte der Amputationswunsch meist deutlich früher auf als in der Pubertät. Die Fetischismus-Hypothese könnte BIID daher nicht vollständig erklä- ren, sondern bestenfalls welche BIID-Betroffene in die Untergruppe der "devotees“ fallen.

\section{Ist der Amputationswunsch wahnhaft?}

Der Drang, sich ein Bein amputieren lassen zu wollen, ohne eine wirklich rationale Begründung hierfür liefern zu können, erinnert an wahnhafte Verhaltensweisen. Im Gegensatz zu Patienten, die unter einem Wahn leiden, sind die BIID-Betroffenen jedoch einsichtig und die Absonderlichkeit ihres Wunsches ist ihnen bewusst. Oft wälzen sie Jahre und Jahrzehnte lang die Argumente. So schrieb der Betroffene-1: „Wenn ich ehrlich zu mir bin, erkenne ich, dass viele Gegenargumente gegen die Realisierung, also Amputation, die ich mir ständig selber vorbete, wie Vorwände sind, teilweise Ängste oder Rücksichtnahme auf andere. Doch scheint mir schon ein Kern da zu sein, der die Amputation nun auch wieder nicht will oder Angst davor hat, so dass ich innerlich durchaus zerrissen bin, worunter ich sehr leide. (...) Bei mir beeinträchtigt BIID das ganze Selbstwertgefühl. Ich traue meinen eigenen Impulsen, Bedürfnissen, Gefühlen und Gedanken nicht so ganz über den Weg, weil ich immer denke: so was Krasses kann ,man' sich doch einfach nicht wünschen!“ An anderem Ort seines Berichts wird das Für und Wieder genau abgewogen: „PRO: ich wäre endlich ich selber; würde mich ganz fühlen; wäre innerlich befreit; sekundär: mein Mut gäbe mir Schwung und mehr Mut. CONTRA: ich wäre behindert: körperlich (langsamer, mehr Kraftaufwand nötig für viele Tätigkeiten, evtl. Phantomschmerzen oder störende Empfindungen, Stumpfschmerzen, schnellerer Verschleiß von Gelenken in Schultern, Armen und Händen, Rückenprobleme, Hilfsmittelbedarf, ...); seelisch (Risiko, dass ich es bereue, dass ich mich schäme, dass ich irgendwie darunter leide, ...); sozial (nicht mehr dazugehören, Außenseiter, immer der Kleinste, schockierender Anblick für manche Leute, Leiden meiner Angehörigen, vor allem der Eltern, sexuell für viele kaum noch attraktiv, ...); wirtschaftlich (was würden meine Kunden sagen? Könnte ich alles in meinen Jobs weiter so machen? Teurere Lebenshaltung, z.B. Verkehr, Reisen, ...); Operationsrisiko; Zeitbedarf für OP, Heilung, Reha, Hilfsmittelbeschaffung (und das immer wieder). In Beispielen: Ich fahre gern Zug und auch ganz kurzfristig - im Rolli in den Zug, das ist vielleicht bei $20 \%$ der Züge und Bahnhöfe leicht, sonst braucht es viel Planung. In einer vollen Kneipe, wo alle stehen, will ich da als einziger im Rolli sitzen, auf Hinternhöhe? (...) Ich möchte nämlich am allerliebsten meine Beine behalten und BIID vergessen." Ein ähnliches Hin und Her wurde auch von den anderen Befragten geäußert: „Bevor ich aber diesen Schritt gehe und ,es' machen lasse, will ich mich vergewissern, ob es nicht noch andere Wege gibt, BIID zurückzudrängen oder zu beseitigen, so dass ich damit leben kann." Oft wird eine Amputation aus Vernunftgründen verschoben. So hatte der Befragte-5 konkrete Planungen, sich den Zeigefinger abzutrennen, musste bei der "Generalprobe“ mit einem Kaninchenschenkel aber feststellen, dass es nicht wie geplant ging und hat die Entfernung bis heute nicht durchgeführt. Der Befragte-8 schob seinen Wunsch auf, als sein Vater verstarb und er die elterliche Firma übernehmen musste. Diese Einsichtsfähigkeit entspricht nicht einem Wahn.

Medikamentöse und psychotherapeutische Behandlung Wie bereits oben erwähnt, existieren bislang nur Arbeiten auf Einzelfallebene über die Frage, ob psychopharmakologische oder psychotherapeutische Behandlung den BIID-Wunsch verringern kann. Der Befragte-1 dieser Studie hatte sich selbst um 
Behandlungsversuche bei einem Arzt und einem Psychotherapeuten bemüht. Nach seiner Aussage habe dies zwar den Druck für einige Zeit reduzieren können, aber das Problem nicht gelöst. Arzt wie auch Therapeut hatten offen und verständnisvoll reagiert und versucht, mit ihm einen anderen Weg als den chirurgischen zu finden: „Ich mache ja seit September eine selbsterfahrungsorientierte Kunsttherapie, mit Elementen der Meditation usw. Das tut mir auch gut! Aber BIID lässt sich davon kaum beeindrucken. Leider!!!“ Ihm wurde empfohlen, es mit der verhaltenstherapeutischen Gedanken-Stopp-Technik zu versuchen. Hierzu schrieb er nach einigen Monaten: „Es klappt eine Weile, aber innerlich baut sich in der Zeit ein zunächst nicht spürbarer, dann aber spürbarer Druck auf, und es kommt wieder, immer wieder, und jedes Mal danach schlimmer als beim letzten ,Hoch'. (...) Und die Gedankenstopptechnik funktioniert auch nicht, wenn ich morgens noch im Halbschlaf sehr intensiv in meinen Beinen die darin ,verborgenen' Stümpfe spüre, ihre genaue Länge, sogar ein bisschen die Form. Da bin ich noch gar nicht fähig zur Gedankenkontrolle.“ Negative Erfahrungen bei seinen Bemühungen um Therapie machte dagegen der Befragte-2: „Vergangenes Jahr war ich bei 2 Psychologen, bei denen ich auf völliges Unverständnis und komplette Unkenntnis stieß.“ Die dritte Person hatte sich in eine Verhaltenstherapie begeben, gleichfalls ohne eine Veränderung des BIID-Wunsches erzielen zu können. Die übrigen Teilnehmer dieser Befragung hatten keinen derartigen Therapieversuch unternommen. Der Befragte-8 lehnte dies auch vehement ab. Nr-9 schrieb hierzu: „Ich habe mich noch nicht getraut. Man will ja nicht als Spinner dastehen. (...) Jedenfalls glaube ich, würde meine Umwelt eine freiwillige Amputation nicht verstehen. Für sie sind Amputierte Behinderte. Für mich nicht unbedingt.“

\section{Diskussion}

BIID gibt weiterhin Rätsel auf. Auf den ersten Blick gibt es Parallelen zu neurologischen Störungen wie z. B. Neglekt oder Asomatognosie. Letztere haben nach einer schweren Hirnschädigung gleichfalls das Gefühl, dass Körperteile nicht zu ihnen gehören. Oliver Sacks [46] beschrieb einen Schlaganfall-Patienten mit Neglekt-Symptomatik, der versuchte sein Bein aus dem Bett zu stoßen, weil er meinte, man habe das tote Bein einer fremden Person zu ihm ins Bett gelegt. Allerdings leiden diese Patienten unter großflächigen Läsionen des parietalen Kortex, sie sind halbseitengelähmt und können das entsprechende Glied nicht spüren. Vergleichbare Schäden weisen BIID-Betroffene definitiv nicht auf. Bayne und Levy [1] haben darauf hingewiesen, dass „Wannabes“ keinerlei Bewegungsschwierigkeiten haben, die bei einer solchen Läsion aber nachweisbar sein müssten. Darüber hinaus planen die meisten später eine Prothese zu benutzen. Dies würden sie nicht tun, wenn das entsprechende Glied gar nicht in ihrem mentalen Körperschema repräsentiert wäre.

In der Arbeit von First [20] wollten 38 der 52 Befragten die Amputation eines Beins und nur 7 die des Arms. Wäre der Amputationswunsch das Ergebnis einer frühkindlichen Hirnfunktionsstörung, müsste man annähernd dieselben Zahlen für Arm- und Bein-Amputation erhalten. Eher müsste es sogar noch mehr Wünsche nach einer Arm-Amputation geben, da das Hirnareal mit der Repräsentation der Arme an der oberen Außenseite des Gehirns liegt und daher eher geschädigt werden kann als das Areal für die Beine, das gut geschützt am Interhemisphärenspalt liegt. Insbesondere der Fakt, dass der Amputationswunsch bei einem Teil der Betroffenen von einer Körperseite auf die andere wechseln kann, spricht nicht dafür, dass hier eine Läsion des somatosensorischen Kortex vorliegt. Interessanterweise findet man eher rationale Begründungen dahingehend, welche Körperseite amputiert werden soll. Dass mehr Bein- als Arm-Amputationen gewünscht werden, könnte möglicherweise daran liegen, dass man ein fehlendes Bein mit Krücken, Prothesen oder Rollstuhl im Alltagsleben besser ausgleichen kann als einen fehlenden Arm. In der Studie von First [20] gaben 44 der 52 Befragten eine spezifische Seite der Amputation an; hiervon wollten nur 12 eine rechtsseitige Amputation, die zu größeren Schwierigkeiten führt (z. B. beim Autofahren). Immerhin 12 der von First Befragten zeigten variable Muster bezüglich der Lokalisation der Amputation. Einige waren offenbar zufrieden damit, irgendein Körperteil zu entfernen, bei anderen wechselte die Präferenz über die Zeit von einem auf andere Körperteile.

Aktuell laufen Untersuchungen mithilfe von fMRI [51], um neuronale Korrelate der BIID zu finden. Die bisherigen Befunde unterstützen aber kaum den Verdacht, dass lediglich ein ZNS-Defekt für den Amputationswunsch verantwortlich ist. Die Betroffenen scheinen eher die „Idealvorstellung“ eines perfekten Körpers zu haben, der einen Arm oder ein Bein zu wenig umfasst. Diese Annahme einer solchen Idealvorstellung einer Behinderung erfährt Unterstützung dadurch, dass es einige verwandte Syndrome gibt. So existieren Menschen, die sich völlig andere Einschränkungen wünschen wie z.B. Blindheit, Taubheit [53] oder sogar Inkontinenz.

Manche der Betroffenen sehen Amputierte als „Helden“, die durch ihre persönliche Stärke trotz der Behinderung ihr Leben meistern. In diesem Sinne überrascht es nicht, dass viele amputierte Personen Sportarten wie Skifahren, Bergsteigen oder Radfahren machen, die man ihnen auf den ersten Blick nicht zutraut [29]. Sportveranstaltungen wie die „Paralympics“ und Sportler wie z. B. Oscar Pistorius zeigen, dass die Fähigkeiten solcher „Behinderter" oft weitaus besser sein können als in der unbehinderten Durchschnittsbevölkerung. Kino-Kultfilme wie „Planet Terror“ (Regie: R. Rodriguez, 2007), in dem die Hauptdarstellerin statt auf einer Beinprothese auf einem Maschinengewehr läuft, unterstützen diesen Trend.

Hieraus stellt sich die Frage, ob die Betroffenen im Grunde unter Minderwertigkeitskomplexen leiden und nun versuchen durch die Bewältigung einer schweren Behinderung der Umwelt zu zeigen, was in ihnen steckt. Evereard [18] stellte einen Patienten vor, der bis zu seinem 44. Lebensjahr noch nie Sexualkontakt hatte und berichtete, dass seine Eltern ihm in der Kindheit verboten hatten, mit den Unterschicht-Kindern auf der Straße zu spielen. Er beobachtete aber häufig einen Jungen mit Holzbein, der in die Kindergruppe integriert war und durch seine Behinderung nicht wirklich beeinträchtigt schien: “...nevertheless I considered him happier than myself and felt that it was not so awful to be without a leg." Evereard vermutete, dass das Holzbein für den Patienten ein Synonym für Glück wurde. Money und Simcoe [38] beschrieben einen Betroffenen, der als Kind dick und unbeholfen war und berichtete, dass vor allem der Sportunterricht die Hölle für ihn gewesen war. Oft hatte er als Kind gedacht, wenn er amputiert wäre, müsste er jetzt nicht über die Hürden laufen. Bruno [11] entwickelte auf der Basis dieser Beschreibungen die Theorie, dass BIID-Betroffene in ihrer Kindheit Ablehnungs-Erfahrungen gemacht hatten und dann beobachteten, dass Behinderte mehr Zuwendung von ihrer Umwelt erhalten. Via Identifizierung könnte es dazu gekommen sein, dass die Betroffenen nun glauben, dieselbe Aufmerksamkeit zu bekommen, wenn sie 
selbst behindert sind. Unsere Daten unterstreichen nicht, dass schwere psychische Störungen wirklich eine Rolle spielen. Von den hier vorgestellten Betroffenen zeigte nur ein einziger Symptome einer erheblichen Depression. Die übrigen waren sowohl testpsychologisch wie auch im persönlichen Kontakt unauffällig; sie hatten durchgängig hohe Ausbildungsabschlüsse, gingen einer geregelten Arbeitstätigkeit nach und die meisten lebten in Partnerschaften.

Die Betroffenen selbst weisen immer wieder auf Parallelen zur Transidentität („gender identity disorder“, GID) hin. Diese Personen haben seit früher Kindheit das Gefühl, in einem Körper mit dem falschen Geschlecht zu leben. Viele versuchen jahrzehntelang damit zurecht zu kommen, etliche heiraten sogar. Oft wird das Verlangen, das äußere Geschlecht an das mentale anzupassen aber irgendwann so groß, dass eine Geschlechtsumwandlung durchgeführt wird. Die Parallelen zu BIID sind nicht von der Hand zu weisen. Allerdings weiß man heute, dass das mentale Geschlecht schon im fötalen Stadium durch Sexualhormone geprägt wird. Durch Krankheiten oder Stress kann es offenbar dazu kommen, dass hier zu hohe bzw. zu niedrige Spiegel dieser Sexualhormone vorhanden sind und das Gehirn nicht entsprechend des real existierenden Chromosomensatzes geprägt wird. GID-Betroffene haben ebenso wie BIID-Betroffene das Gefühl, im falschen Körper zu leben; ohne aber die äußeren Genitalien direkt abzulehnen. Zum Beispiel hassen männliche Transidente, die eine Umwandlung zur Frau wünschen, ihr Geschlechtsteil nicht. Sie empfinden es lediglich nicht als Teil ihrer wahren Identität. Der wesentliche Unterschied zwischen GID und BIID ist, dass es keine Hormone gibt, die ein Gehirn auf "Einbeinigkeit“ prägen.

Aller Wahrscheinlichkeit nach ist BIID ebenso wenig eine einheitliche Störung wie Schizophrenie, Sucht, Depression oder Neurose, sondern man muss mindestens 3 Achsen trennen: 1. Stärke der hirnorganischen Dysfunktion, 2. Ausprägung von psychischen Komponenten (z.B. sekundärer Krankheitsgewinn) und 3. Ausmaß der sexuellen Komponente. $\mathrm{Zu}$ trennen wären hier wie auch bei vielen anderen psychischen Störungen 2 Bereiche von ursächlichen Einflussfaktoren:

1. Neurobiologische Grundlage. Die starke emotionale Reaktion, die von den Betroffenen in der Kindheit auf den Anblick eines Amputierten geschildert wurde, lässt vermuten, dass es nach dem verhaltensbiologischen Schlüssel-Schloss-Prinzip eine Hirnveränderung gegeben hat, die in einem sehr frühen Stadium der Entwicklung stattfand. Die Betroffenen sehen in der Kindheit einen Amputierten, dies erschreckt sie aber nicht, sondern passt in ihr persönliches Selbstbild und führt zu einem Aha!-Erlebnis. Bei BIID-Betroffenen mit Sensibilitätsdefiziten des betroffenen Körperteils könnte diese Dysfunktion im parietalen somatosensorischen Hirnareal liegen. Diese Läsion könnte allerdings nicht erklären, warum der Amputationswunsch von einer Seite auf die andere wechseln kann. Denkbar wäre auch eine Dysfunktion im thalamischen Bereich. Thalamo-kortiko-thalamische Systeme sind in die mentale Körperrepräsentation stark involviert [30]. Hier könnte das Konzept einer „Einbeinigkeit“ ohne konkrete Lateralisierung eher verankert sein, was den Wechsel des Amputationswunsches erklären könnte.

2. Psychosoziale Einflüsse: Die Identifikation mit einer behinderten Person in einer sensiblen Phase der Kindheit scheint die meisten BIID-Personen stark beeinflusst $\mathrm{zu}$ haben $[8,11,18,20,22,31,37,39,49]$. Hier spielen diverse psychologische Einflussfaktoren eine Rolle. Einige Betroffene erleben im Sinne des Freud'schen Konzepts des sekundären Krankheitsge- winnes, dass Behinderte mehr Liebe von der Umwelt erhalten; andere bewundern die Leistungsfähigkeit behinderter Idole. Unerforscht ist, in wie weit durch diese Idealisierung eigene Minderwertigkeitsgefühle kompensiert werden sollen. Zumindest einige Betroffene äußern diesbezüglich, dass sie der Umwelt beweisen wollen, gerade mit einer Behinderung dennoch selbstständig lebensfähig zu sein. Im Bereich psychosozialer Einflüsse wäre im Sinne einer fetischistischen Konditionierung auch BIID mit einer sexuellen Komponente anzusiedeln. Beispiele wie die oben zitierte Geschichte des Mannes, der seinen ersten Orgasmus ausgerechnet beim Pretending bekam, untermauern, dass die Möglichkeit besteht, auch Amputationen mit sexuellen Gefühlen zu besetzen.

Die Einstellung der Betroffenen lässt sich vielleicht am besten durch folgende kurze Geschichte herauskristallisieren, die seit einiger Zeit auf einschlägigen Internet-Seiten Betroffener kursiert: „Wenn ich Morgen früh eine gute Fee auf der Fensterbank sitzen finde, die mich fragt, ob ich nicht gern- Simsalabim - kurz und problemlos meine Amputation haben möchte, komplett mit Alibi und allem Drum und Dran - nun, dann freue ich mich natürlich riesig. Wenn nun aber daneben gleich noch eine gute Fee sitzt, die mich fragt, ob ich nicht vielleicht einfach gern rundum zufrieden mit meinem Körper sein möchte und von BIID nie wieder im Leben irgendwas hören oder fühlen ... nun - dann schubse ich die erste Fee doch geradewegs aus dem Fenster und nehme das zweite Angebot. Von allen Möglichkeiten möchte ich am allerliebsten geistig und körperlich absolut gesund und unbehindert sein." (www.forumromanum.de)

\section{Take Home Message}

Als Fazit für die Praxis lässt sich sicherlich ziehen, Menschen mit Amputationswunsch nicht vorschnell als psychotisch einzustufen. Die Betroffenen selbst leiden beträchtlich unter ihrem Wunsch und wissen durchaus, dass dieser nicht normal ist. Oft wägen sie jahrzehntelang das Für und Wider ab. Unklar ist, wie man den Betroffenen am wirksamsten helfen kann. Zur Lösung dieser Frage hat sich im Herbst 2008 eine überregionale BIID-Kommission gebildet mit Vertretern aus Österreich (Roswith Roth, Graz), der Schweiz (Peter Brugger, Zürich) und Deutschland (Aglaja Stirn, Frankfurt und Erich Kasten, Lübeck), die als Ansprechpartner zur Verfügung stehen.

\section{Literatur}

1 Bayne T, Levy N. Amputees by choice: Body Integrity Identity Disorder and the ethics of amputation. Journal of Applied Psychology 2005; 22 (1): $75-85$

2 Becker P. TIPI - Trierer Integriertes Persönlichkeitsinventar. Goettingen: Hogrefe, 2000

3 Beckford-Ball J. The amputation of healthy limbs is not an option. British Journal of Nursing 2000; 9 (4): 188

4 Beckmann D, Brähler E, Richter HE. Gießen-Test. Goettingen: Hogrefe, 1990

5 Bensler J, Paauw D. Apotemnophilia masquerading as medical morbidity. Southern Medical Journal 2003; 96: 674 - 676

6 Berger BD, Lehrmann JA, Larson $G$ et al. Nonpsychotic, nonparaphilic self-amputation and the internet. Comprehensive Psychiatry 2005; 46 (5): $380-383$

7 Blanke O, Morgenthaler FD, Brugger P et al. Preliminary evidence for a fronto-parietal dysfunction in able-bodied participants with desire for limb amputation. Journal of Neuropsychology [ahead of print], 
8 Braam AJ, Visser S, Cath DC et al. Investigation oft the syndrome of Apotemnophilia and course of a cognitive-behavioral therapy. Psychopathology 2006; 39: $32-37$

9 Brenner I. Upper-extremity self-amputation in a case of dissociative identity disorder. Journal of Clinical Psychiatry 1999; 60: 705

10 Bridy A. Confounding extremities: Surgery at the medico-ethical limits of self-modification. The Journal of Law, Medicine \& Ethics 2004; 32 (1): $148-158$

11 Bruno RI. Devotees, pretenders and wannabes: Two cases of factitious disability disorder. Journal of Sexuality and Disability 1997; 15 (4): $243-260$

12 Coons PM, Ascher-Svanum H, Bellis K. Self-amputation of the female breast. Psychosomatics 1986; 27: 667f

13 Coons PM. Self-Amputation of the Breasts by a male with schizotypal personality disorder. Hosp. Community Psychiatry 1992; 43: 175 - 176

14 Cuen I, Benkoil D. Britain mulls surgery for healthy limbs: Surgical questions. ABC-news.com, February 4, 2000

15 Dotinga $R$. Out on a Limb, Salon.com (Aug. 29, 2000) http:// dir.salon.com /health /feature /2000 /08 /29 /amputation /index.html,

16 Dyer C. Surgeon amputated healthy legs. British Medical Journal 2000; 320: 332

17 Elliott C. To be mad. The Atlantic Monthly. Dec. 2000: 73-84

18 Evereard $W$. A case of apotemnophilia: a handicap as sexual preference. American Journal of Psychotherapy 1983; 37: 285-293

19 Fahrenberg J. FBL - Freiburger Beschwerdeliste. Goettingen: Hogrefe, 1994

20 First MB. Desire for amputation of a limb: paraphilia, psychosis or a new type of identity disorder. Psychological Medicine 2004; 34: 1 - 10

21 Franke GH. SCL-90R - Die Symptom-Checklist von Derogatis. Goettingen: Hogrefe, 2002

22 Furth G, Smith R. Apotemnophilia: Information, questions, answers, and recommendations about self-demand amputation. 1st Books Library: Bloomington, IN, 2000

23 Furth GM, Smith R, Kubler-Ross E. Amputee Identity Disorder: Information, questions, answers, and recommendations about self-demand amputation. Autorhouse, 2000

24 Gregson I. The Apotemnophile: An amputees perspective. Amputeeonline.com, March 6., 2000

25 Griffith ER, Fleischmann RB, Hohmann GW. et al. Sexual dysfunctions associated with physical disabilities. Archives Physical and Medical Rehabilitation 1975; 56: 8

26 Hall D, Lawwon B, Wilson I. Commons hallucinations and self-amputation of the penis and hand during a first psychotic break. Journal of Clinical Psychiatry 1981; 42: 322 - 324

27 Hautzinger $M$, Bailer $M$, Worall $H$ et al. Beck Depressions Inventar. Goettingen: Hogrefe, 1995

28 Hoermann H, Moog W. Rosenzweig Picture Frustration Test. Goettingen: Hogrefe, 1954

29 Horn F. A life for a limb. Social Work Today 2003; 3: 16-19

30 Jones EG. The Thalamus. Cambridge Uni. Press, 2007

31 Kasten E. Body-Modification. Psychologische und medizinische Aspekte. München: Reinhardt, 2006
32 Kasten E, Stirn A. Wechselnder Amputationswunsch von links nach rechts bei Body Integrity Identity Disorder (BIID). Zeitschrift für Psychiatrie, Psychotherapie und Psychologie 2009; 57 (1): 55ff

33 Kasten E. Pattern of personality in subjects with Body Integrity Identity Disorder (BIID). (submitted),

34 Large M, Babidge N, Andrews D et al. Major self-mutilation in the first episode of psychosis. Schizophrenia Bulletin, 2008 [ahead of print]

35 London LS, Caprio FS. Sexual deviations. Washington: Linaere Press, 1950

36 MacKenzie R, Cox S. Transableism, disability and paternalism in public health ethics: taxonomies, identity disorders and persistent unexplained physical symptoms. International Journal of Law 2007; 2 (4): $363-375$

37 Money J, Jobaris R, Furth G. Apotemnophilia: two cases of self-demand amputation as paraphilia. Journal of Sex Research 1977; 13: 115 - 125

38 Money J, Simcoe KW. Acrotomophilia, sex and disability: New concepts and case report. Sexuality and Disability 1986; 7: 43-50

39 Müller S. Dieses Bein will ich nicht. Gehirn \& Geist 2007; 7-8: 66 - 70

40 Müller S. Body Integrity Identity Disorder (BIID) - Ist der Amputationswunsch eine autonome Entscheidung oder Ausdruck einer neuropsychologischen Störung? In: Groß D, Müller S, Steinmetz J (eds). Normal - anders - krank. Berlin: Medizinische Wissenschaftliche Verlagsgesellschaft, 2007

41 Nitschmann K. Chirurgie für die Seele? Zeitschrift für die gesamte Strafrechtswissenschaft 2007; 119: 547-592

42 Parsons J, Brown W, Sirota A. Inappropriate amputational requests. Psychosomatics 1981; 22: 822-823

43 Pollmann A. Ein Recht auf Unversehrtheit? Skizze einer Phänomenologie moralischer Integritätsverletzung. In: S Walt, C Menke (eds). Die Unversehrtheit des Körpers. Frankfurt a. M.: Campus, 2007: 214- 236

44 Riddle GC. Amputees and devotees: Made for each other? New York, Irvington Publisher, 1998

45 Ryan CJ. Out on a limb: The ethical management of Body Integrity Identity Disorder. Neuroethics [ahead of print],

46 Sacks O. Der Mann, der seine Frau mit einem Hut verwechselte. Reinbeck: Rowohlt, 1998

47 Schlozman S. Upper extremity self-amputation and replantation: 2 case reports and a review of the literature. Journal of Clinical Psychiatry 1998; 59: $681-686$

48 Smith RC. Amputee identity disorder and related paraphilias. Psychiatry $2004 ; 3$ (8): $27-30$

49 Sorene ED, Heras-Palou C, Burke FD. Self-amputation of a healthy hand: A case of body integrity identity disorder. Journal of hand surgery 2006; 31 (6): $593-595$

50 Stewart $D$, Lowrey $M$. Replantation surgery following self-inflicted amputation. Canadian Journal of Psychiatry 1980; 25: $143-150$

51 Stirn A, Thiel A, Skoruppa S et al. Body integrity identity disorder - characteristics and neural correlates. European Psychiatry 2008; 23 (2): S191

52 Tavcar R, Dernovsek M, Zvan V. Self-amputation of left hand: a case report. Journal of Clinical Psychiatry 1999; 60: 793 - 794

53 Veale D. A Compelling Desire for Deafness. Journal of Deaf Studies and Deaf Education 2006; 11 (3): 369-372 